\title{
A left-sided neck mass (2010: 8a)
}

(C) European Society of Radiology 2010

\section{History:}

A 45-year-old man with a long-standing history of a painless mass in the left submandibular region presented to the Head and Neck Surgery Clinic due to progressive increase of the mass over the past 2 months. No associated inflammatory signs were present and there was no history of salivary colic or any prior intervention in the submandibular glands. His past medical history was remarkable for snoring with no signs of obstructive sleep apnoea. His past surgical history was unremarkable. Computed tomography (CT) was performed at a outside facility reporting a left submandibular gland mass and the patient was then sent to the Cancer Institute for further management.

Non-enhanced CT (Fig. 1a, b) were performed.

What is the diagnosis?

Readers are invited to supply one possible diagnosis via electronic means to: robert.hermans@uzleuven.be

The subject of the email should include 'Interpretation Corner' and the number given above (e.g. Interpretation Corner 2010: 8a). They should include their name, title, a

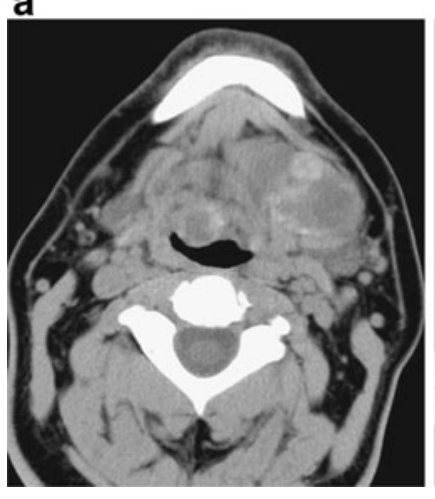

b

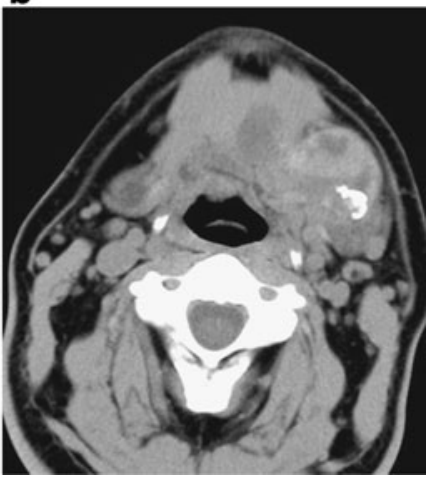

Fig. 1 Plain transverse CT sections through the sublingual/ submandibular space $(\mathbf{a}, \mathbf{b})$

address, FAX and phone number. Deadline: one clear calendar month from distribution date. The names of the first 25 radiologists submitting the correct diagnosis will be published (only one from any individual centre and none from the host institution!). Three months after the initial publication of the case history, the authors will publish the final diagnosis. 